\title{
An unusual cause for periodic limb paralysis; Gitelman syndrome
}

\author{
Jayasekara JMS, Palangasinghe DR, Bodinayake CK \\ Professorial Medical Unit, Teaching Hospital, Karapitiya, Galle, Sri Lanka. \\ Correspondence: Dr. Swarnamali Jayasekara \\ e-mail: swarnamalij2005@gmail.com \\ https://orcid.org/0000-0002-6378-2463
}

\section{Introduction}

Gitelman syndrome (GS) is an autosomal recessively inherited salt loosing tubulopathy with a prevalence of $1-10$ per 40,000 people $(1,2)$. The prevalence of GS is higher in Asia than other countries (2). GS is characterized by hypokalaemic metabolic alkalosis, hypomagnesaemia and hypocalciuria (3). It is typically seen in late childhood or adulthood. Symptoms are related to the degree of electrolyte disturbance. Cramps of the limbs are present among almost all and fatigability, polyuria, polydipsia, chondrocalcinosis are the other reported symptoms $(1,4)$. Clinical manifestations are less pronounced in heterozygotes (1).

Here we report a case of GS presented with a hypokalaemic periodic quadriparesis.

\section{Case report}

A 15 year-old previously healthy school boy noticed bilateral upper limb and lower limb weakness when he got up in the morning. Two days prior he experienced similar kind of weakness which resolved spontaneously. Weakness was not ascending and he denied dysphagia or breathing difficulty. His urinary and bowel habits were normal and there was no history of trauma.

Examination revealed flaccid quadriplegia with muscle power Grade 3/5 in upper limbs and Grade $2 / 5$ in lower limbs. Power of neck muscles was normal and cranial nerve examination was unremarkable. All the reflexes were diminished and plantar response was normal. There was no sensory impairment. He was neither dyspnoeic nor tachypnoeic. Single breath count was more than 20 . His pulse rate was 100/ minute and blood pressure was $135 / 65 \mathrm{mmHg}$. Respiratory system and abdominal examinations were unremarkable.
Evaluation revealed serum $\mathrm{K}^{+} 2.1 \mathrm{mmol} / \mathrm{L}(3.5$ - 5.3), serum corrected calcium $2.59 \mathrm{mmol} / \mathrm{L}(2.1-2.57)$, serum magnesium $0.68 \mathrm{mmol} / \mathrm{L}(0.66-1.07), \mathrm{pH}$ 7.42 with $\mathrm{HCO}_{3} 28 \mathrm{mmol} / \mathrm{L}, 24$ hour urinary calcium excretion $0.2 \mathrm{mmol} /$ day $(2.0-7.5 \mathrm{mmol} /$ day) urinary excretion of sodium $126 \mathrm{mmol} / \mathrm{L}$, urinary potassium excretion $112 \mathrm{mmol} / \mathrm{L}$ and urinary chloride excretion $140 \mathrm{mmol} / \mathrm{L}$, supine aldosterone $343.2 \mathrm{pg} / \mathrm{mL}(49.3$ - 175) and supine renin 36.1 $\mathrm{pg} / \mathrm{mL}(2.7$ - 32.6). Parental screening revealed asymptomatic hypokalaemia. (mother $-3.4 \mathrm{mmol} / \mathrm{L}$, father- $3.1 \mathrm{mmol} / \mathrm{L}$ )

Intravenous potassium chloride $(\mathrm{KCl})$ was commenced to correct the serum potassium deficit followed by oral $\mathrm{KCl}$ one tablet twice daily and he showed a remarkable response. Later he was started on spironolactone and $\mathrm{KCl}$ was tailed off. He was asymptomatic on subsequent clinic visits and serum potassium remained normal.

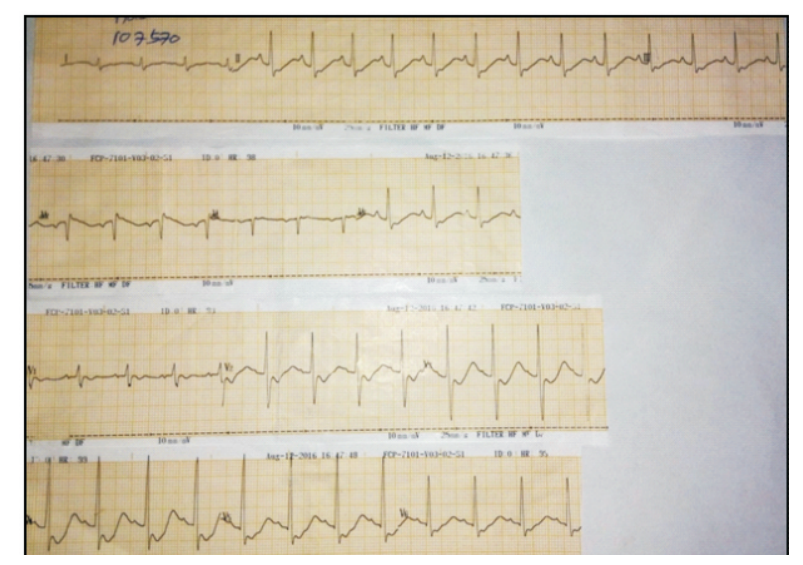

Figure 1: ECG on admission showing $U$ waves and prolonged QT interval suggestive of hypokalaemia 


\section{Discussion}

GS was first described in 1966, and its genetic basis was elucidated 30 years later. GS is unarguably the most frequent inherited tubulopathy and autosomal recessive in inheritance $(5,6)$. In the great majority GS is caused by mutations in the SLC12A3 gene, which encodes the renal thiazide-sensitive sodiumchloride co-transporter (TS-NCC) that is specifically expressed in the apical membrane of cells in the distal convoluted tubule (DCT) (7).

Reduced NCC activity mimics the effects of persistent thiazide diuretic action, which include volume contraction, reduced or normal blood pressure, increased renin activity and aldosterone levels, renal potassium wasting and hypokalemia, renal magnesium wasting and hypomagnesaemia, and reduced urinary calcium excretion (1).

Impaired sodium chloride reabsorption leads to mild volume depletion and activation of the renin-angiotensin-aldosterone system (1). The combination of secondary hyperaldosteronism and increased distal flow and sodium delivery enhances potassium and hydrogen secretion at the connecting and collecting tubules leading to hypokalaemia and metabolic alkalosis (1). Hypocalciuria occurs due to loss of activity of TS-NCC which increases tubular reabsorption (3).

The diagnosis of GS is based on the clinical symptoms, biochemical abnormalities and largely one of exclusion with combination of characteristic set of metabolic abnormalities which includes hypokalaemia, hypomagnesaemia, metabolic alkalosis, secondary hyperaldesteronism, reduced urinary calcium excretion and increased urinary excretion of sodium and chloride $(7,1)$. Other tests, such as genetic testing and measurement of the change in fractional excretion of chloride in response to loop and thiazide diuretics, are not widely performed (1).

Our patient exhibited clinical and almost all the laboratory criteria with the asymptomatic hypokalemia in both parents supporting autosomal recessive inheritance. Tubular defect of the GS cannot be corrected. Therefore treatment is life-long and is aimed at minimizing the effects of the secondary increases in renin and aldosterone production and at correcting the volume deficit and electrolyte abnormalities. Genetic counseling is utmost important as the recurrence risk for parents with an affected child is $25 \%$ (7).

In general, the long-term prognosis is excellent and progression to renal insufficiency is extremely rare in GS (7).

\section{Conclusions}

Gitelman syndrome is one of the rare causes for hypokaleamia which is treatable and has excellent long term outcome. We achieved a good clinical and biochemical response with oral $\mathrm{KCl}$ followed by spironolactone.

\section{References}

1. UpToDate 21.6. Bartter and Gitelman syndromes.

2. Zhonghua Nei Ke Za Zhi. Expert consensus for the diagnosis and treatment of patients with Gitelman syndrome; Gitelman Syndrome collaborative study group; 2017 Sep 1; 56(9): 712-6. [PubMed].

3. DR Mhasde, S Gautam, RR Zore, Shoeb Nadeem, PV Chole, SP Ganeshpure. Gitelman Syndrome. Journal of the Association of Physicians of India, March 2009; 57: [Google Scholar].

4. Ayşe Şeker Koçkara, Ferhan Candan, Can Hüzmeli, Mansur Kayataş, Demet Alaygut. Gitelman syndrome associated with chondrocalcinosis. Journal of Renal Failure; 10 Sep 2013.

5. Gitelman HJ, Graham JB, Welt LG. A new familial disorder characterised by hypokalaemia and hypomagnesemia. Trans Assoc Am Physicians 1966, 79: 221-35.

6. Farhan Raza Mubashar Sultan, Khola Qamar, Ali Jawad, Ali Jawa. Gitelman syndrome manifesting in early childhood and leading to delayed puberty: a case report. Journal of Medical Case Reports, 2 October 2012. [Google Scholar].

7. Nine VAM Knoers, Elena N Levtchenko. Gitelman syndrome. Orphanet Journal of Rare Diseases, 30 July 2008. [Google Scholar].

8. Anne Blanchard, Detlef Bockenhauer, Davide Bolignano, Lorenzo A. Calò, Etienne Cosyns, Olivier Devuyst, David H. Ellison, Fiona E. Karet Frankl, Nine V.A.M. Knoers, Martin Konrad, Shih-HuaLin, RosaVargas-Poussou. Gitelman syndrome: consensus and guidance from a Kidney Disease: Improving Global Outcomes (KDIGO) Controversies Conference; Meeting Report, January 2017, Pages 24-33. [Google Scholar]. 